\title{
Teleodontologia e Teleducação: Design de estratégias de visualização avançada para a supervisão docente em ambiente de telemonitoramento e teleconsulta
}

\author{
Rafael M. Toscano \\ Laboratório de Aplicações de \\ Vídeo Digital, UFPB \\ João Pessoa - PB, Brasil \\ rafaeltoscano@lavid.ufpb.br \\ Fernanda C. Almeida \\ Faculdade de Odontologia - USP \\ (FOSUP) \\ São Paulo - SP, Brasil \\ fernandacsa@usp;br
}

\author{
Dandara Estrela \\ Laboratório de Aplicações de \\ Vídeo Digital, UFPB \\ João Pessoa - PB, Brasil \\ dandara.estrela@lavid.ufpb.br
}

Mary Caroline S. Macedo

Faculdade de Odontologia - USP

(FOSUP)

São Paulo - SP, Brasil

mary@usp.br

\author{
Cicero I. da Silva \\ Design Educacional - TEDE \\ UNIFESP \\ São Paulo - SP, Brasil \\ cicero.silva@unifesp.br
}

\author{
Guido Lemos \\ Labratório de Aplicações de Vídeo \\ Digital, UFPB \\ João Pessoa - PB, Brasil \\ guido@lavid.ufpb.br
}

\begin{abstract}
This article proposes an advanced interaction and visualization strategy for teacher supervision in a telemonitoring and teleconsultation environment based on video-based services. For the development of the proposal, a case study was carried out with the USP School of Dentistry. The proposal considers issues such as the hierarchy of supervision, request for help and communication, classification of priority of service; control of information flow and marking of contextual attributes such as time and type of service. Finally, it is believed that the presented prostitution is a potential solution both in the scenario of Teledentistry and in other contexts of education and health.
\end{abstract}

\section{KEYWORDS}

Telehealth, Teledentistry, HCI, Advanced visualization, Video

\section{Introdução}

A telessaúde provê acesso a procedimentos de saúde por meio de tecnologias síncronas e assíncronas, teleodontologia, por sua vez, pode ser compreendida como a provisão remota de atendimento odontológico, por meio de tecnologias da informação, em vez de contato pessoal e direto com qualquer paciente envolvido [1]. Uma das principais estratégias é a utilização de serviços de vídeo para a comunicação entre profissionais e profissional-paciente. Estes serviços incluem desde avaliações remotas, relatos prégravados, video bot's, consultorias, formação em saúde a realização de atendimentos e procedimentos por videochamadas.

No contexto do ensino e da educação permanente em saúde (ensino-serviço), a telessaúde em geral, e a teleodontologia em particular, impõe desafios adicionais à sua implantação, já que são necessários controles rigorosos dos fluxos de trabalho

In: VII Workshop "O Futuro da Videocolaboração” (WCT-Video 2020), São Luís, Brasil. Anais Estendidos do Simpósio Brasileiro de Sistemas Multimídia e Web (WebMedia). Porto Alegre: Sociedade Brasileira de Computação, 2020.

() 2020 SBC - Sociedade Brasileira de Computação.

ISSN: $2596-1683$ presenciais/remotos e a capacidade de acompanhamento, supervisão e gestão dos serviços prestados [2]. Esse processo chamado de preceptoria, ou supervisão, ocorre tanto nas atividades diárias de uma clínica ou hospital quanto em ambientes de formação de profissionais em saúde e é vital para garantir segurança aos usuários do serviço e qualidade de aprendizado aos alunos.

O supervisor está na instituição para dar suporte aos profissionais de menor experiência. Em alguns momentos ele acompanha o passo a passo de atividades e em outros é consultado para auxiliar em situações específicas. Além de supervisionar a atuação de outros profissionais, o supervisor pode realizar procedimentos modelos, isto é, executar atividades em que ele irá exemplificar um processo, rotina ou conhecimento. Outra característica da supervisão é a distribuição da responsabilidade de supervisão. Por exemplo, ao invés do Médico/ dentista/ psicólogo/professor (supervisor) supervisionar e orientar todos os demais profissionais, ele ocupa-se em orientar os residentes de maior experiência, que por sua vez orientam residentes de menor experiência até os internos.

Apesar dos diversos avanços dos VAS (do inglês, Video as Services) na área de saúde, a atividade de supervisão profissional não está completamente amparada nas discussões e soluções atuais. Esta realidade envolve desde desafios computacionais de processamento e rede até processos e estratégias para a visualização e interação com usuários de múltiplas videochamadas simultâneas.

A criação de uma estratégia ou projeto de interação para viabilizar a supervisão de múltiplos atendimentos síncronos, no contexto da telessaúde, especialmente nos ambientes de ensino, é um desafio que está alinhado tanto às grandes discussões na IHC, [3] quanto ao cenário da vídeocolaboração [4].

Esta pesquisa teve o Design Science Research (DSR) como processo metodológico [5]. Da compreensão do contexto externo (literatura) e das características do ambiente interno (FOUSP) surge a classe de problemas desse estudo. A partir desses dois elementos identificou-se como tópicos a serem trabalhados: 1. a 
configuração de um processo de interação capaz de viabilizar a Hierarquia de Supervisão; 2. pedido de ajuda e comunicação entre profissionais/alunos e supervisores ao longo de teleatendimentos; 3. classificação de prioridade de atendimento; 4. controle de fluxo de informações; 5 . atributos contextuais: tempo (duração e início de um atendimento), perfil ou tipologia do atendimento, etapas do atendimento (ex: identificação, queixa principal, prescrição de medicamento e requisição exames).

Para compreender melhor este cenário de visualização avançada, foi realizado um estudo com as ações de teleodontologia da clínica escola da Faculdade de Odontologia da Universidade de São Paulo (FOUSP). Esta pesquisa é fruto dos avanços do projeto Video For Health (V4H) apresentado em edições anteriores deste workshop [6]. Além das questões de segurança, preservação o V4H tem avançado na criação de um serviço baseado em vídeo para a realização de atendimentos na área de saúde através da parceria e colaboração técnica entre LAVID-UFPB, FOUSP, Telessaúde-SP, Wisecare e RNP.

\section{Estado da arte}

Pesquisas anteriores identificaram uma lacuna na literatura teórica e metodológica na área de Interação HumanoComputador (HCI) para auxiliar no desenvolvimento de software para sistemas audiovisuais, aplicações multimídia ou ambientes hipermídia baseados em vídeo [7]. As produções identificadas na revisão da literatura foram sustentadas principalmente por métodos tradicionais de IHC, como o User-Centered Design. Do ponto de vista dos Estudos de Mídia, também houve poucos avanços nos últimos anos.

Conforme destacado no contexto externo dessa pesquisa não foram encontrados outros sistemas de videochamada com foco na supervisão. Por conta disso, foi realizado um comparativo entre as versões mais atuais dos sistemas: Google Meet, Facetime, Whereby, Discord, Zoom e Skype. De modo geral todos os sistemas apresentam diferentes formas de visualização dos participantes de uma videochamada, além do padrão face-to-face.

Nesse contexto destacam-se as opções de mosaico, grid flexível, container redimensionável, miniatura da chamada PIP (do inglês, Picture in Picture), que pode ser vista até mesmo quando o usuário minimiza o navegador e utiliza outras aplicações. Além dessas opções de exibição do conteúdo as diferentes formas de exibição de chat privado e a criação de salas simultâneas são recursos que, mesmo projetados para outros propósitos, revelam-se como potenciais soluções ao contexto da supervisão.

\section{Visualização avançada para a supervisão na teleodontologia}

Para o Design Science Research (DSR), após a definição da classe de problemas o próximo passo da pesquisa é a proposição de um artefato com o intuito de alcançar a resolução de tais itens.
A discussão que fundamenta essa proposta de visualização avançada de teleatendimentos é a arquitetura de design colaborativo para imersão temporal e espacial [8]. A partir dessa discussão é possível compreender que a supervisão de múltiplos atendimentos, é, antes de tudo, a visualização de múltiplos eventos de áudio e vídeo.

\subsection{Visualização simultânea de atendimentos}

A visualização e a interação com múltiplos eventos de áudio e vídeo pode ocorrer essencialmente de dois modos: espacial e temporal.

A visão espacial consiste em um arranjo dos containers de vídeo que permite ao usuário (Supervisor) observar, avaliar, hierarquizar e interferir em todos os eventos simultaneamente. A composição visual pode variar desde um grid pré-determinado, listas até mosaicos flexíveis que são dinâmicos de acordo com critérios pré-estabelecidos pelo sistema ou supervisor. Apesar dos diversos fluxos de vídeo estarem sendo reproduzidos simultaneamente, apenas um fluxo de áudio pode ser percebido por vez sem prejudicar a compreensão ou mesmo aumentar a carga mental dos indivíduos. Desse modo, o acesso ao áudio dos atendimentos pode ser feito por seleção do supervisor, tempo ou qualquer critério pré-estabelecido.

Já a visão temporal consiste no foco da visualização de um container por vez. Essa observação pode ocupar a tela inteira ou parte dela, estar condicionada a um tempo pré-determinado (ex: ver cada atendimento por trinta segundos) ou ser alternada por ação do próprio usuário (supervisor).

A supervisão de múltiplos atendimentos simultâneos, no contexto da telessaúde, vai além da sobreposição de eventos de áudio e vídeo, já que a atividade que está sendo realizada através do serviço de vídeo têm características ou atributos de grande relevância para o supervisor. Esses elementos podem atuar como indicadores para facilitar e categorizar a exibição dos containers de vídeo de acordo com critérios relevantes ao serviço prestado. São exemplos de atributos contextuais: tempo (duração e início de um atendimento), perfil ou tipologia do atendimento, etapas do atendimento (ex: identificação, queixa principal, prescrição de medicamento e requisição exames).

\subsection{Perfis e modos de interação com o sistema}

Tendo em vista o contexto da supervisão, identificado na literatura e no ambiente interno da clínica escola de odontologia, percebeu-se a existência de diversos perfis que podem ser assumidos ao longo de um atendimento.

Supervisor Chefe: Profissional de maior experiência, que pode atuar como líder do departamento, serviço ou procedimento.

Supervisor: Profissional intermediário que atua sob a supervisão do Supervisor Chefe e acompanha/supervisiona os profissionais em formação.

Profissional: Profissional em formação e/ou que está realizando o atendimento direto com o paciente.

Paciente: Indivíduo que está sendo atendido. 
Teleodontologia e Teleducação: Design de estratégias

Convidado: Indivíduo sem vínculo com a instituição que está participando pontualmente do atendimento.

Os papéis descritos podem ser assumidos tanto por pessoas distintas quanto pelo mesmo indivíduo em determinadas situações; por exemplo, a estrutura de algumas instituições permite que o supervisor chefe seja também o supervisor e/ou atue como profissional, durante a realização de um procedimento que ainda não tenha sido realizado na clínica.

No contexto da clínica de odontologia da FOUSP, um professor (Supervisor-Chefe) é responsável por dez alunos de pós graduação (Supervisor). Cada um desses dez alunos orienta mais dez alunos de graduação (Profissional). Cada profissional conduz o atendimento ao paciente, e, em alguns casos, há também a presença de um convidado.

No dia a dia, o profissional (P) é acompanhado pelo supervisor (S). Em situações pontuais, o profissional $(\mathrm{P})$ poderá solicitar a ajuda e a presença do seu supervisor direto (S) no atendimento, assim como, do supervisor chefe (SC) da clínica.

Cada perfil identificado tem prioridades e requisitos diferentes diante do sistema. Por conta disso, propõe-se uma interface diferente para cada um.

Dashboard: painel com acesso aos dados gerais dos atendimentos, disponibilidade dos profissionais, chat público e privado, lista de pedidos de ajuda, histórico de atendimentos e revisão e assinatura de documentos criados pelos profissionais e supervisores.

Sala de supervisão: módulo do sistema que permite ao Supervisor (S) gerenciar as atividades do seu grupo de profissionais, solicitações de ajuda, histórico de atendimentos, visualização de atributos contextuais e documentos pendentes.

Atendimento: módulo do sistema que concentra a videochamada entre profissionais, pacientes e convidados. O profissional poderá ver sua agenda de atendimentos, marcar ou classificar os atributos contextuais definidos pela instituição (ex: classificação de risco e perfil do atendimento), chat privado com supervisores, solicitação de ajuda durante o atendimento, visualizar atendimentos realizados. Já o paciente poderá acessar o atendimento, utilizar o chat com o profissional e enviar um convite para convidados.

\subsection{Dinâmicas de interação}

A gestão dos múltiplos eventos simultâneos é o principal desafio do design de interação de um sistema com foco na supervisão profissional. Para lidar com esse cenário a solução proposta foi a criação dos perfis, interfaces e recursos de modo a estabelecer a hierarquia de supervisão ao longo das videochamadas. O SC tem acesso a um painel com dados gerais do sistema e a todos os grupos de supervisão que estão atuando no momento.

Cada Grupo de supervisão é composto por um Supervisor (S) e dez Profissionais (P). Para a clínica cada grupo de supervisão dispõe sempre dos mesmos participantes. Apesar dessa configuração, a proposta também pode ser aplicada em contextos de maior flexibilidade em que tanto o supervisor quanto os profissionais serão agrupados no esquema de plantão.

A partir do painel destacado na figura 1, o Supervisor Chefe (SC) acompanha tanto o número de atendimentos que estão em
Anais Estendidos do WebMedia'2020, São Luís, Brasil

andamento, quanto sua classificação de risco. Ao detalhar tais opções o SC poderá assumir o papel de Supervisor (S) e observar ou participar de um atendimento, iniciar uma nova videochamada temporária com o profissional (sem a presença do paciente) ou mesmo conversar com o profissional em um chat privado.

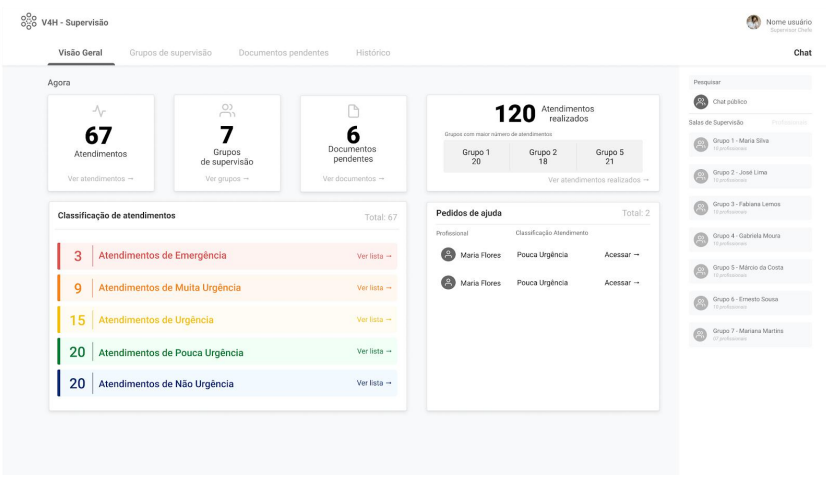

Figura 1: Dashboard Supervisor Chefe

O pedido de ajuda e comunicação entre os usuários do sistema está presente de forma distinta para cada papel. Para o Supervisor Chefe (SC), o canal de comunicação permite que o mesmo envie mensagens que serão recebidas para todos da instituição, como uma lista de transmissão, mas também permite mensagens privadas. O Supervisor (S) no entanto, tem acesso à conversas privadas apenas com o grupo de alunos que ele supervisiona. Para o caso do profissional, existe apenas um chat entre ele e o seu supervisor.

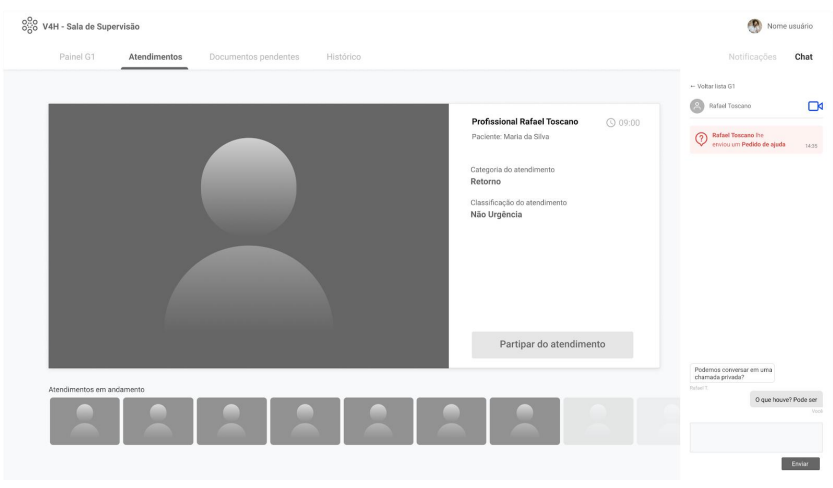

Figura 2: Supervisor acompanhando atendimento ao mesmo tempo que interage pelo chat com o profissional

O pedido de ajuda está presente durante a realização do atendimento. Essa ajuda pode ocorrer diretamente em um chat privado, pela participação direta do supervisor na sala ou mesmo por uma consultoria em uma videochamada temporária.

Além da visualização espacial, para todos os atendimentos simultâneos (figura 3), e espacial (figura 2) a compreensão dos supervisores é aprimorada pela utilização de atributos contextuais ao serviço prestado. A classificação de risco nesse protótipo representa um exemplo de critério contextual para o 
estudo de caso. A depender do contexto ou serviço de saúde outros critérios poderão ser adotados.

Ao longo do atendimento o profissional terá a opção de classificar seu atendimento de acordo com o risco avaliado. Essa classificação será percebida pelo supervisor como uma categoria ou etiqueta atrelada a cada um dos atendimentos. Na figura 3 é possível ver a exibição dos atendimentos de um grupo de supervisão a partir das categorias de risco dos atendimentos. Tal escolha permitirá tanto ao supervisor dar ênfase aos eventos críticos quanto reduzir a necessidade de banda para utilizar o serviço já que o número de atendimentos reproduzidos simultaneamente é controlado pela visualização das categorias.

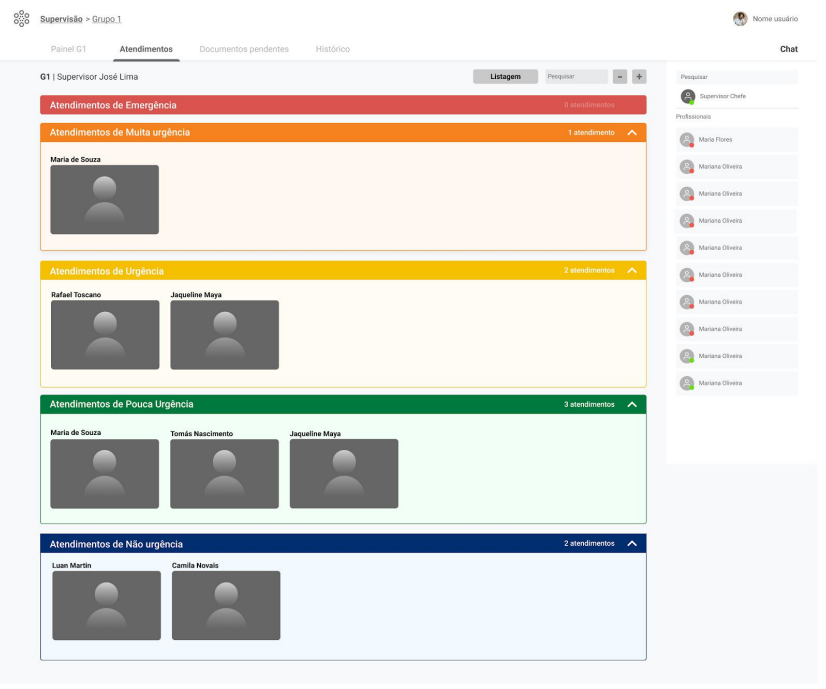

Figura 3: Visualização dos atendimentos através de categorias contextuais ao serviço.

Sobre o uso desses indicadores para etapas ou momentos de um atendimento, este pode ser acionado manualmente pelo Profissional, realizado através de um assistente para o atendimento ou mesmo por extração em áudio de palavras chaves ditas pelos participantes.

\section{Lições aprendidas}

Para validar tais conceitos foram desenvolvidos cinco protótipos de alta fidelidade envolvendo áudio, vídeo e multiusuários. Esses protótipos foram testados por 3 profissionais que atuam na área da saúde em encontros distintos que totalizaram na execução de 20 tarefas por usuário. A participação desses profissionais teve o objetivo de avaliar noções de utilidade, facilidade e percepção de valor.

A pesquisa ainda encontra-se em andamento, com a perspectiva de realização de novos testes com mais usuários, todavia, até o presente momento foram apreendidas as seguintes lições: (a) a organização da hierarquia através das estratégias propostas apresentou-se como uma abordagem clara, intuitiva e viável para a implementação final do serviço; (b) O desafio inicial, de IHC e rede, de monitorar 100 atendimentos simultâneamente foi contornado de forma satisfatória, inclusive, percebeu-se que com a filtragem e organização dos atendimentos o número de eventos simultâneos cai expressivamente para os supervisores o que aumenta a viabilidade e aplicabilidade da proposta; (c) a estrutura de supervisão somada aos recursos de segurança, gravação e preservação dos atendimentos em blockchain, ofertados pelo $\mathrm{V} 4 \mathrm{H}$, aumentam a segurança jurídica e profissional da qualidade do serviço prestado. Os impactos destes elementos ficam a cargo de estudos futuros, na continuação desta pesquisa.

\section{Visão de futuro}

Diante da literatura analisada e da experiência dos pesquisadores deste trabalho percebeu-se que os desafios e tendências de pesquisas nessa área estão alinhadas à pelo menos três tópicos, são eles: (a) a criação de novos métodos, padrões ou convenções de design para integrar e viabilizar a comunicação de múltiplos usuários (um para um e um para muitos) a partir de dispositivos em contextos de acesso e uso diversificado; (b) a criação ou evolução de protocolos, serviços ou camadas de software robustos que atuem como agregadores e orquestradores de fluxos de áudio e vídeo a partir de tipos distintos de rede ou sinal; (c) a criação de rotinas, métricas e atributos contextuais para avaliar, classificar e indexar os registros de áudio e vídeo dos serviços de saúde como forma de documentação legal, dados clínicos, acompanhamento da saúde ou mesmo a criação de bases de dados para o ensino e formação em saúde.

\section{ACKNOWLEDGMENTS}

Agradecemos a toda a equipe do projeto Video For Health (V4H) pelo apoio técnico e científico ao desenvolvimento desta pesquisa.

\section{REFERENCES}

[1] Khan, S. A., \& Omar, H. (2013). Teledentistry in practice: literature review. Telemedicine and e-Health, 19(7), 565-567.

[2] Granja, C., Janssen, W., \& Johansen, M. A. (2018). Factors determining the success and failure of eHealth interventions: systematic review of the literature. Journal of medical Internet research, 20(5), e10235.

[3] Stephanidis, C., Salvendy, G., Antona, M., Chen, J. Y., Dong, J., Duffy, V. G., ... \& Guo, Y. (2019). Seven HCI grand challenges. International Journal of Human-Computer Interaction, 35(14), 1229-1269.

[4] Roesler, V., Carissimi, A., Ciuffo, L., \& Lopes, P. (2018). Visão de Futuro Comitê Técnico de Vídeocolaboração (CT-Vídeo).

[5] Dresch, A., Lacerda, D. P., \& Júnior, J. A. V. A. (2015). Design science research: método de pesquisa para avanço da ciência e tecnologia. Bookman Editora.

[6] Sousa, D., Souza, G., Silva, C., \& Castro, G. (2019). Vídeo Síncrono com Preservação Digital e Registro Distribuído como um Serviço para Telessaúde. In Anais Estendidos do XXV Simpósio Brasileiro de Sistemas Multimídia e Web, (pp. 175-178). Porto Alegre: SBC.

[7] Toscano R.M., de Souza H.B.A.M., da Silva Filho S.G., Noleto J.D., Becker V. (2019) HCI Methods and Practices for Audiovisual Systems and Their Potential Contribution to Universal Design for Learning: A Systematic Literature Review. In: Antona M., Stephanidis C. (eds) Universal Access in HumanComputer Interaction. Theory, Methods and Tools. HCII 2019. Lecture Notes in Computer Science, vol 11572. Springer, Cham

[8] TOSCANO, Rafael et al. Arquitetura de design colaborativo para imersão temporal e espacial em vídeos de altíssimas resoluções e HFR. O futuro da videocolaboração: perspectivas. Porto Alegre, RS: Sociedade Brasileira de Computação (SBC), p. 13-53, 2017. 